Author's reply: Professor David highlights important points in relation to the trial of cognitive therapy for the prevention of psychosis. He asks for clarification regarding the exclusion of two participants for having been psychotic at inception, but only reporting this on second contact with an assessor. This was certainly what happened, and following this the research assistants were instructed to ask all participants about this. This was not in the original protocol for the study, as such an event was unexpected (although, with hindsight, maybe it should not have been). It did seem reasonable to exclude these participants, especially given that the study is the first of its kind (clearly future studies should address this issue in the protocol).

$\mathrm{He}$ also raises the issue of randomisation. The procedure for randomisation is very clearly outlined within the original paper and the difference in gender rates was due to chance. It is true that this method resulted in more of the treatment group being female, which is an indicator of better outcome for such a population; however, the method also resulted in the treatment group having a higher proportion of people who were unemployed and a significantly higher level of baseline positive symptoms, both of which would predict poorer outcomes for the treatment group. It is also worth noting that gender was utilised as a covariate in all analyses regarding transition that were reported.

Professor David has identified two important issues that can only be clarified by replication of the results of this study with a more rigorous protocol and an alternative method of randomisation; we would agree that such research is required to determine whether the preventive effects of cognitive therapy with people at ultrahigh risk of developing psychosis are generalisable and robust.

A. P. Morrison Department of Psychology, University of Manchester, Coupland, Manchester MI3 9PL, UK

\section{Guided self-change for bulimia}

Bower \& Gilbody (2005) raised questions about stepped care in psychological therapies to which I would like to provide some answers. Our treatment evaluation study of guided self-change for bulimia nervosa incorporated a self-care manual and investigated acceptability, drop-out rate, 'extra treatment' and longer-term outcome (Thiels et al, 1998a, 2003). We did not dare to offer the manual only as a first step to Germans spoilt by a healthcare system with excellent provision of long-term psychotherapy. Instead we compared 8 fortnightly sessions plus a self-care manual (guided self-change) with 16 weekly individual sessions of cognitive-behavioural therapy (CBT). There were no significant differences between therapies regarding dropout rate, general satisfaction with treatment and views regarding the usefulness of the therapies.

A journalist who wanted to write about our study in the weekly newspaper Die Zeit met with strong resistance. Although we published the 6-month follow-up results in the American Journal of Psychiatry (Thiels et al, 1998b, quoted by Bower \& Gilbody, 2005) the head of the science section of Die Zeit did not believe that such low therapist input could work. Some psychiatrists in the hospital where the journalist's husband worked feared for their jobs.

Bower \& Gilbody (2005) state that 'research on the patient acceptability assumption would need to access the views of a number of different populations...' We invited family physicians, psychiatrists, gynaecologists and various counselling services to refer those who complained of symptoms suggestive of bulimia nervosa (the clinical picture of which was briefly described) (Thiels et al, 1998b). An article about the service in a local newspaper led to several self-referrals. Unfortunately, we did not compare acceptability according to the source of referral and thus can only report the overall acceptability.

Follow-up by personal interview using expert and self-rated instruments was carried out a mean of 54.2 months (s.d.=5.8) after the end of therapy. Significant improvements were achieved or maintained in both groups on the main outcome measures. These included eating disorder symptoms from expert ratings (Eating Disorder Examination sub-scores: overeating, vomiting, dietary restraint, shape and weight concern), self-report (Bulimic Investigatory Test, Edinburgh) and a global five-point severity scale. Improvement was also seen on the subsidiary outcome variables Beck Depression Inventory, SelfConcept Questionnaire and knowledge of nutrition, weight and shape. During the week before follow-up $66.7 \%$ of the guided self-change group and $61.5 \%$ of the CBT group had not binged, vomited or misused laxatives.
A considerable proportion in both groups had extensive further psychological treatment, mainly for their eating disorder. The majority of these had not done well during initial treatment. An analysis of covariance showed a significant interaction between treatment group and additional treatment between the 6-month and the 4-year follow-up. Cross-tabulation showed that this difference was due to the fact that three of the five in the CBT group with additional treatment between the two follow-up assessments improved more than the eight in the CBT group without additional therapy $\left(\chi^{2}=6.24 ; \quad\right.$ d.f. $=1$; $P<0.035)$. However, the six in the guided self-change group who received additional treatment made as little progress as the seven who did not $\left(\chi^{2}=0.26\right.$; d.f. $=1$; $P<1.00)$. Four out of 12 individuals received additional treatment from their study therapist, the other eight received additional treatment elsewhere. The question is whether those who were allocated to guided self-change would not have recovered in the course of 4 years with any therapy or whether they might have done better with more therapist contact from the beginning of treatment.

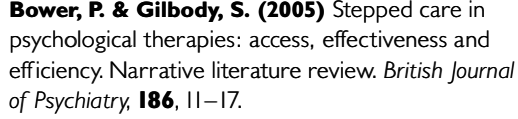
efficiency. Narrative literature review. British Journal of Psychiatry, 186, II-17.

Thiels, C., Schmidt, U., Treasure, J. L., et al (1998a)

Wie wirksam und akzeptabel ist ein

Selbstbehandlungsmanual mit begleitender

Kurztherapie bei Bulimia nervosa? Nervenarzt, 69 427-436.

Thiels, C., Schmidt, U., Treasure, J. L., et al (1998b) Guided self change for bulimia nervosa incorporating a self-care manual. American Journal of Psychiatry, I55. 947-953.

Thiels, C., Schmidt, U., Treasure, J. L., et al (2003) Four year follow-up of guided self change for bulimia nervosa. Eating and Weight Disorders, 8, 212-217.

C.Thiels Department of Social Sciences, University of Applied Sciences, Kurt-SchmacherStrasse 6, Bielefeld, D-336I5 Germany

\section{What is a traumatic event?}

Olff et al (2005) sampled farmers during a foot and mouth epidemic, concluding that half whose livestock were culled had 'post-traumatic stress at levels requiring professional help', and 'the high prevalence of post-traumatic stress symptoms is an underestimation of the real levels of psychopathology' (p. 166). What was the 'traumatic event' implicated in these 